\title{
Pupil Detection Algorithm Based on Feature Extraction for Eye Gaze
}

\author{
S.Deivanayagi, V.G.Nandhini Sri, P.Kalai Priya, G.Aarthi
}

\begin{abstract}
Exact real-time pupil tracking is an essential step in a live eye gaze. Since pupil centre is a base point's reference, eye centre localization is essential for many applications. In this research, we extract pupil eye features exactly within different intensity levels of eye images, mostly with localization of determined interest objects and where the human is looking for. Since it's a digital world and digital transformation, everything is becoming virtual. Hence this concept has a huge scope in e-learning, class room training and analyzing human behaviour. This research covers eye tracking technology to track and analyze the learners' behavior and emotion on e-learning platform like level of attention and tiredness. Harr's cascade classifier was used to first locate the eye's area, and once found and support vector machine (SVM) for classification with the trained datasets. We also include the state of emotions, facial landmarks of the salient patches on face image using automated learning-free facial landmark detection technique.Experimental results help in developing learner eye gaze detection in system using Pycharm and hardware output using Raspberry Pi. In Raspberry Pi is given with the input image captured using external webcam and based on the engagement level of the learner content 1 or 2 would be displayed in the Raspbian OS environment.
\end{abstract}

Key Words: Image processing, SVM, Harr's Cascade.

\section{INTRODUCTION}

In a virtual learning world, learners can lose motivation and concentration very easily. Our research is based on studying learner's behavior on an online learning platform to create a system able to analyze the learners based on their behavior, emotion and listening to educational content to their needs.

Eye tracking is one of the techniques for recording eye movements. This technology is used to measure eye positions and eye movement in many fields such as psychology, psycholinguistics, ergonomics and e-learning. This paper introduces the use of eye tracking technology to track and analyze the learners' behavior and emotion on e-learning platform like level of attention and tiredness.

In e-learning, it is necessary to create more effective interaction between the educational content and learners. In particular, increasing motivation by stimulating learners' interest is very much important. Users' eyes can be a significant source of information to analyze learners' behavior and listening to class. Eye movements provide an indication of learner interest and focus of attention. Movement of eyes provides useful feedback to personalize learning interactions which can help in effective teaching. With a study of eye movement, learners may be more motivated.

Revised Manuscript Received on July 22, 2019.

S.Deivanayagi, Associate Professor,Department of ECE,Sri Sairam Institute of Technology

V.G.Nandhini Sri, P.Kalai Priya, G.Aarthi Final year students,

Department of ECE,Sri Sairam Institute of Technology

\section{RELATED WORKS:}

"AUTOMATED WHEELCHAIR" can be regulated by theEYE-BALL movement by utilizing the concepts of Image Processing and other guiding technologies [1].

High accuracy of face recognition, detection of facial parts such as eyes, nose, and mouth is achieved by 2D Hough transformation for detecting circle of unknown radius in which, first it generates 2D parameter space (xc, yc) using the gradient of grayscale through obtaining the radius of circle $r$ for each local maximum in the (xc, yc) space. The next step is eye detection using Support Vector Machine (SVM). At last, pairs of eyes satisfying predefined conditions are generated and ordered by sum of the likelihood of both eyes.[2]

An eye tracking system helps in tracking the movement of the eyes to know exactly where the person is looking and for how long they stare at. The suitable devices for eye movement acquiring and software algorithms are chosen as per the application requirements[7]. Some vendors have invested in eye tracking technology. But their solutions are focused on commercial remote camera-based eye-tracker systems for which the light source and camera are permanently affixed to a monitor which is considered as one of the demerits of the system.[3]

The automatic eye detection technique is subsequently validated using FRGC 1.0 database. The result of validation shows that our eye detector has an overall $94.5 \%$ eye detection rate, with the detected eyes very close to the manually provided eye positions. [4]

Three different algorithms were used for eye pupil location and testing. This algorithm efficiency comparison was based on human face images taken from the BioIDdatabase. In this case human face images were acquired by a webcam and processed in a real-time system [5].

For images with low resolution, computer vision community due to noise, shadows, occlusions, pose variations, eye blinks, etc., is used and a two-stage algorithm is proposed for iris centre localization[8]. A fast convolution based approach is used for obtaining the coarse location of Iris Centre (IC) and IC is further refined in next stage using boundary tracing and ellipse fitting. The algorithm has been evaluated in public databases like BioID, Gi4E[6].

To improve cursor stability, eye pupil center was filtered with Gaussian filter to remove the spikes[9].

The viability of autonomous public eye trackers as both data-gatherers and public exhibits is proposed in this research[10].

In automotive applications, integrated power electronic systems for automotive electronics gives a solution to

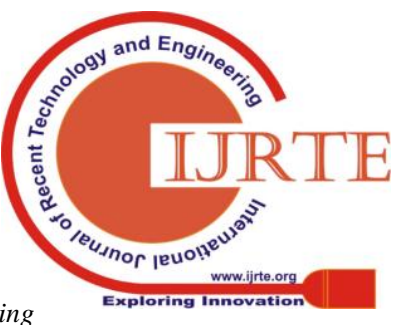
\& Sciences Publication 


\section{Pupil Detection Algorithm Based on Feature Extraction for Eye Gaze}

future challenges of electronic printed circuit boards[11].

\section{METHODOLOGY:}

After detecting the objects in the facial area, our proposed method is placing a sliding window in the middle area of the face image, not from the top left corner as was done by the conventional haar cascade. Before, images have been through grayscaling process and change the pixel value into the integral image, determination ROI of eye, than in this area performed calculation to get features value of eye.

In our research, an exact real-time pupil tracking is done in a live eye gaze. Since pupil centre is a base point's reference, exact eye centre localization is essential for finding out the listeners' eye movement while conducting the class. In this research, we extract pupil eye features exactly within different intensity levels of eye images, mostly with localization of determined eye pupil and where the human is looking for. Since it's a digital world and digital transformation, everything is becoming virtual now-a-days. Hence this concept has a huge scope in e-learning, class room training and at an extent of analyzing human behaviour. This research covers main process like Eye Ball movement detection, mentality and mood Recognition of Human beings. Harr's cascade classifier was first used to locate the eye's area, and once it is found, a classification with the trained datasets was done by using Support vector machine (SVM).

Automated learning-free facial landmark detection technique is used to obtain the state of emotions, facial landmarks of the salient patches on face image and the software used is Python in the platform of Pycharm.

\section{WORKING PRINCIPLE:}

The block diagram of the model is shown in the fig.1. The external webcam captures the eye movement and it is given to Raspberry Pi which undertakes the process of images such as Pre-processing, Segmentation and Eye Ball detection via feature extraction.

i) PRE PROCESSING:

The face image captured by webcam is processed to remodel color image into grayscale image is the pre-processing technique underwent firstly. It is necessary to transform the RGB image into grayscale image by taking the average of the RGB values and the average value of that can be obtained as grayscale is given by the mathematical expression as,

Grayscale $=(\mathrm{R}+\mathrm{G}+\mathrm{B}) / 3$

The Face detection and eye region localization is carried out by obtaining the knowledge of the position and pose of the face is an essential factor in determining the point of gaze. Detection of the face helps in obtaining listeners' eye ball movement detection. This reduces the false positive rate and the computation time. Haar-like feature based method is used for face detection because of its higher accuracy and faster execution time.

\section{ii) SEGMENTATION:}

Segmentation of pupil of eye is a challenging, yet important, and a step towards the analysis of digital images. The rate, automation, accuracy and robustness of the segmentation methods often have a large impact on the performance of the application.
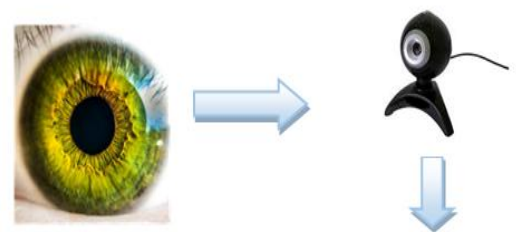

EYES

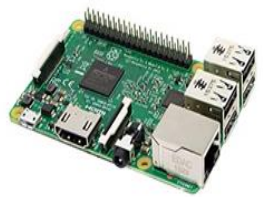

Pre-processing

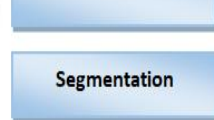

Eyeball detection

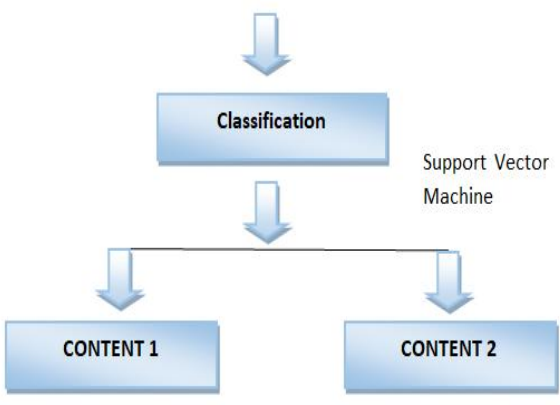

Fig. 1 Block diagram

The Random Walker algorithm is a recently-developed multi-label image segmentation method that helps the user to select background and objects' seeds. We can quickly determine the un-labeled pixel by using small number of pixels with predefined labels which will first reach one of the pre-labeled pixels. The greatest probability is calculated by assigning each pixel to the label. Hence, image segmentation is obtained.

Multi-label image segmentation method that allows the user to select background and objects' centre of listeners' eye. Given a small number of pixels with predefined labels, one can quickly determine the probability that a random walker starting at each unlabeled pixel will first reach one of the pre-labeled pixels.

By assigning each pixel to the label for which the greatest probability is calculated from which image segmentation is obtained. 


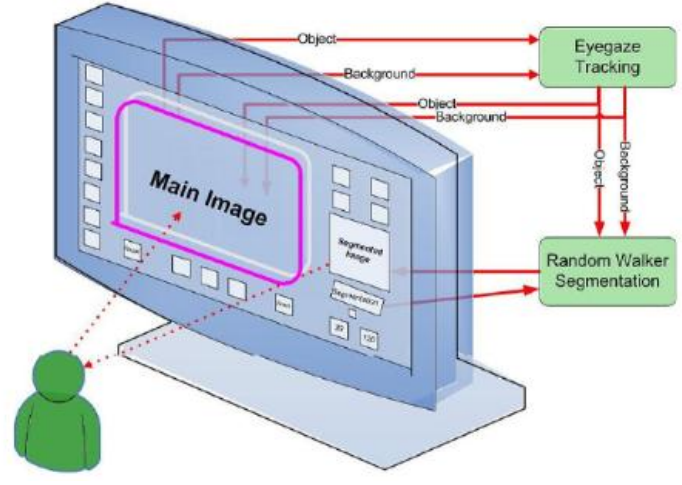

Fig.Se

\section{gmentation}

iii) EYE BALL DETECTION:

Initial step for finding the eye ball is tracking the centre of Iris performed by Harr's cascade in a video sequence. The search region for iris detection can be reduced with the tracking approach. Once the IC is detected, the point can be tracked in subsequent frames easily.

In the next step, open and closure of the eye is been detected by IC localization algorithm that may return false positives when the eyes are closed. Thresholds on the peak magnitude are used to eliminate false positives. However, the quality of peak may deteriorate in conditions such as low contrast, image noise and motion blur. The accuracy of the algorithm may fall in these conditions, and hence Machine Learning (ML) based approach is used to classify the eye states as open or close.

\section{FEATURE EXTRACTION:}

Haar Cascade:Insignificant variations in movements and blinking of eye which are formed by the appearance of inner eye corner Therefore, this research was designed to use inner eye corners as reference points for gaze tracking. The eye corners can be find easily in the eye ROI. The position of gaze is easily detected by using the vector connecting eye corners and iris centre.

In this research, we make use of a sliding window and haar features. The detection window starts from the center of the image which located the area of eye to reduce the processing time and errors in eye detection.

Feature extraction was performed to detect the Eye Gaze. This paper explicitly shows that the use of inner eye corners as reference points for gaze tracking. The eye corners can be located easily in the eye region of interest. The vectors connecting eye corners and iris centre can be used to calculate eye gaze position accurately.

Mainly, Support vector machines (SVMs) seek to define a linear boundary between classes such that the margin of separation between samples from different classes that lie next to each other is maximized. Classification by SVMs is applicable only with data from each class near the decision boundary, called support vectors. Support vectors lie on the margin and carry all the desired information about the classification problem.

\section{RESULTS AND DISCUSSIONS:}

Fig. 2 shows gray scale conversion in which the RGB color image has been converted to gray scale image as the first step namely, pre-processing in the Image processing. Fig. 3 shows face detection technique for localization of eye specifically.
Fig. 4 shows segmentation of the pupil of the eye and in the Fig. 5 eye ball detection result is shown by SVM ML technique.

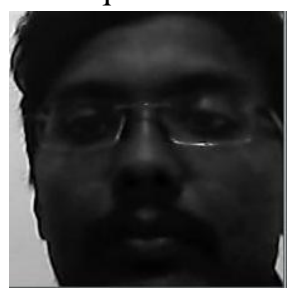

detection

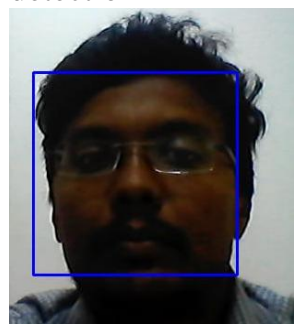

Fig. 4.Segmentation

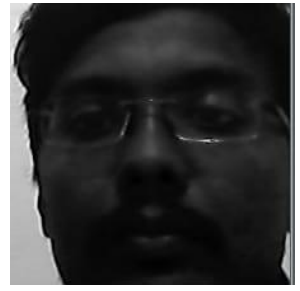

Fig.2.Face

Fig.3.GrayScaling

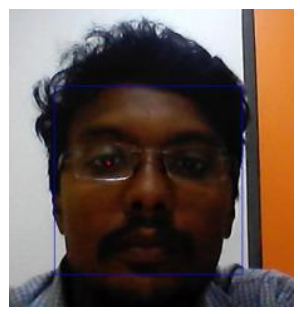

Fig. 5.Iris detection
The image has been captured by using a webcam that has sent to Raspberry Pi. In which the Image processing has been done such as Pre-processing, Segmentation, Eye ball detection using Feature extraction performed by Support Vector Machine and Machine Learning Technique.

\section{CONCLUSION:}

Eye-movement analysis does appear to be a promising new tool for evaluating learners' behavior and emotions. This technology can provide many benefits to e-learning, such as facilitating adaptive and personalized learning. In a couple of years the rapid technical progress may lead to provide accurate eye tracking systems. This is a current area, which has huge scope and applications can be extended upto the users' requirements.

\section{REFERENCES}

1. Parthpancholi, Jaimeet Patel, Saumil Ajmera, "Wheel Chair movement using Eye Ball Detection", International Journal of Advance Engineering and Development (IJAERD) Volume 1,Issue 5,May 2014.

2. Yasu Taka Ito,WataruOhyama, Wakabayashi,Fumitaka Kimura, "Detection of Eyes by circular hough transform and histogram of gradient", Proceedings of the 21st International Conference on Pattern Recognition (ICPR2012), Nov. 2012

3. Robert Gabriel Lupu\& Florina ungureanu, "Survey of Eye tracking methods and applications", UniversitateaTehnică "Gheorghe Asachi" Technical University of Iaşi, Faculty of Automatic Control and Computer Engineering,August 2013

4. PengWang,MathewB.Green,Qiang ji \&James Wayman, "Automatic Eye Detection and its validation" ,Proceedings of the 2005 IEEE Computer Society Conference on Computer Vision and Pattern Recognition (CVPR'05),2005

5. Michal Ciesla,"Eye Pupil location using Webcam", Department of Physics, Astronomy and Applied Computer Science, Jagiellonian University, Poland, 2010.

6. Anjith George, "Fast and accurate algorithm for Eye localization for gaze tracking in low resolution images", IET Computer vision, 2016.

7. D. Li, D. Parkhurst, "Open-source software for real-time visible spectrum eye tracking", 2nd Conference on Communication by Gaze Interaction (COGAIN), 2006, Sept. 4-5, 2006, Turin, Italy.

8. A. Al-Rahayfen, M. Faezipour, "Eye Tracking and Head Movement Detection: A State-of-Art Survey",

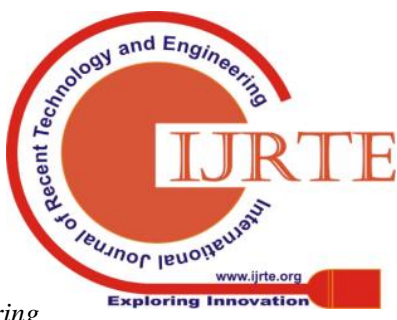

Published By: Engineering \& Sciences Publication 


\section{Pupil Detection Algorithm Based on Feature Extraction for Eye Gaze}

Rehabilitaion Devices and Systems, IEEE Journal of Translational Engineering in Health and Medicine, vol. 1, 2013, 10.1109/JTEHM.2013.2289879, 2013.

9. R. Ranguram, J.M. Frahm, M. Pollefeys, "A comparative analysis of RANSAC techniques leading to adaptive real-time random sample consesnsus", Computer Vision (ECCV), Springer Berlin Heidelberg, pp. 500-513, 2008.

10. Sheena D., Borah B., "Compensation for some second-order effects to improve eye position measurements", Eye movements: Cognition and visual perception, pp. 257-268, 1981, Hillsdale, Nj: Erlbaum.

11. A. Drumea, P. Svasta, "Designing low cost embedded systems with ethernet connectivity", 17th International Symposium for Design and Technology in Electronic Packaging (SIITME2011), 2011, pp.217-220. 